UD was completed. A near infra-red camera was used to evaluate ICG perfusion of anastomoses (ileum-ileum, right and left ureter with small bowel and colostomy or colo-rectal sides of anastomosis) few second after ICG injection.

Result(s)* Fifteen patients were included in the study. No patient reported adverse reactions to ICG injection. Only 3/15 patients $(20.0 \%)$ had an optimal ICG perfusion $(+++)$ in all anastomoses. The remaining $12(80.0 \%)$ patients had at least one ICG deficit; the most common ICG deficit was on the left ureter: $3(20.0 \%)$ versus $1(6.7 \%)$ patient had no ICG perfusion (-) on the left versus right ureter, respectively $(p=0.598) .8 / 15 \quad(53.3 \%)$ and $6 / 15(40.0 \%)$ patients experienced $\geq$ grade 330 -day early and late postoperative complications, respectively. Of these, two patients had early and one had late postoperative complications directly related to poor perfusion of anastomosis (UD leak, ileum-ileum leak and benign ureteric stricture): all these cases had a sub-optimal intraoperative ICG perfusion.

Conclusion* The use of ICG to intra-operatively assess the anastomoses perfusion at time of pelvic exenteration for gynecologic malignancy is a feasible and safe technique. The different vascularization of anastomotic stumps may be related to anatomical sites and to previous radiation treatment. This approach could be of support in selecting patients at higher risk of complications, who may need personalized follow up.

\section{DOSE-DENSE NACT FOLLOWED BY CCRT IN LOCALLY ADVANCED CERVICAL CANCER: FEASIBILITY AND SAFETY}

${ }^{1} \mathrm{G}$ Parpinel ${ }^{*},{ }^{2} \mathrm{M}$ Buffa, ${ }^{3} \mathrm{JDI}$ Muzio, ${ }^{4} \mathrm{~A}$ Peruzzo Cornetto, ${ }^{5} \mathrm{C}$ Palladino, ${ }^{2} \mathrm{ME}$ Laudani, ${ }^{2} \mathrm{M}$ Barboni, ${ }^{2} \mathrm{E}$ Peirano, ${ }^{2} \mathrm{M}$ Girimonte, ${ }^{4} \mathrm{E}$ Madon, ${ }^{2} \mathrm{P}$ Zola. ${ }^{1}$ University of Turin, Surgical Sciences, Turin, Italy; ${ }^{2}$ University of Turin, Surgical Sciences, Italy; ${ }^{3}$ Città della Salute e della Scienza di Torino, Oncology and Radiation Oncology, Italy; ${ }^{4}$ Città della Salute e della Scienza di Torino, Medical Physics, Italy; ${ }^{5}$ University of Turin, Oncology and Radiation Oncology, Italy

\subsection{6/ijgc-2021-ESG0.25}

Introduction/Background* First-line treatment for locally advanced cervical cancer (LACC) is concurrent platinum chemoradiation therapy (CCRT) followed by cervico-vaginal brachytherapy (BT). Neoadjuvant chemotherapy (NACT) followed by $\mathrm{CCRT}+\mathrm{BT}$ has been proposed as an alternative scheme, but its feasibility is still investigational. The aim of this study was to evaluate safety and efficacy of this treatment.

Methodology In our Institution 30 patients with LACC have been treated between 2016-19. They received 6 cycles of weekly NACT with Carboplatin AUC 2 and Paclitaxel 80mg/ $\mathrm{mq}$, followed by CCRT (pelvic EBRT (45Gy) weekly Cisplatin $40 \mathrm{mg} / \mathrm{mq}$ followed by cervico-vaginal BT-HDR (10Gy).

Primary endpoints were 3-year overall survival (OS) and progression-free survival (PFS) while secondary endpoints were safety and toxicity.

Result(s)* The most frequent histological type was squamous cell carcinoma (80\%) and G3-grading (66,7\%).

9/30 patients had FIGO III stage. Radiological complete response (CR) after NACT was 3,3\% while partial response (PR) was $86,6 \%$; only 1 patient had progressive disease (PD).

21 patients $(70 \%)$ received more than 4 cycles of concurrent Cisplatin during EBRT, while 8 received less than 4 cycles.
After a median follow-up of 36.7 months 3-year OS and PFS values were $71.8 \%$ and $65.2 \%$, respectively. Patients with higher values of haemoglobin pre-CCRT (i.e. $>10 \mathrm{~g} / \mathrm{dl}$ ) reported a superior 3 -year OS value (i.e. $70 \%, \mathrm{n}=25$ ) vs $50 \%$ for patients with $<10 \mathrm{~g} / \mathrm{dl}(\mathrm{n}=5)$.

Local and lymph-node recurrence occurred in 30\% and $23 \%$ of patients while distant-metastasis in $10 \%$ of patients.

Only 1 patient experienced G3 anaemia after NACT while 3 cases of G3 haematological toxicity after CCRT+BT-HDR were observed. One patient had G3 neurotoxicity after NACT and 3 patients experienced G3 nausea and diarrhoea after CCRT+BT-HDR.

Conclusion* In our study NACT followed by CCRT+BT resulted to be a feasible treatment. Our data are consistent with the published literature in term of feasibility and safety and the NACT could by synergic with CCRT in the treatment of LACC.

\section{THE CASE OF CERVICAL CANCER IB3 WITH MIELOTOXITY AFTER 2 CYCLE OF CHEMOTADIOTHERAPY TREATED WITH RADICAL HYSTERECTOMY AS THE LAST METHOD OF CHOICE}

E Milnerowicz-Nabzdyk*, J Tomiczek-Szwiec, K Nowak, M Kalus. PZOZ Opolskie Centrum Onkologii im. prof. Tadeusza Koszarowskiego, Opole, Poland

\subsection{6/ijgc-2021-ESG0.26}

Introduction/Background* Patients with stage IB3 cervical cancer are usually qualified for radiochemotherapy. However, sometimes ther is a high toxicity of this treatment, which lead us to look for non-standard soltions. Nevertheless, we know that a combination of radical surgery, radiotherapy and chemotherapy is also burdened with a high toxicity.

Methodology Radical hysterectomy type C Querleu-Morrow and pelvic and para-aortic lymphadenectomy was performed with laparoscopy - as a minimally invasive procedure.

Result(s)* The case of a 27 year old lady with cervical cancer FIGO IB3 is qualified for chemoradiotherapy (Histopathology: Cervical adenocarcinoma G2). She had received $28 \mathrm{f}$ Grey and one cycle of cisplatin $(70 \mathrm{mg})$. The treatment was postponed because of huge myelotoxicity. As no other choice, she was qualified for radical hysterectomy type C Querleu-Morrow and pelvic - para-aortic lymphadenectomy. Radical hysterectomy was performed with laparoscopy - as a minimally invasive procedure that is less traumating for depressing immune system. Postoperative histopathology: cervix -chronic inflammation. There were observed no neoplastic changes in the uterus, fallopian tubes, ovaries, pelvic and para-aortic lymph nodes. One month after surgery there was observed massive vaginal fistula, massive inflamatory changes were visible in cystoscopy. Two months after surgery -bilateral hydronephrosis, both in double $\mathrm{J}$ ureteral stent were inserted. 5 months after surgery no hydronephrosis was detected, both double J stents from the ureters were removed. Next there were observed episodes of high urosepsis with bilateral hydronephrosis, finally 7 months after surgery - double J stends to both ureters were necessary.

Conclusion* We are conscious that finally, retransplantation of the ureters is necessary otherwise the patient will be obliged to continuous exchange of the double J stands in both ureters and the episodes of the urosepsis would reply - but if the urologic surgery is needed it could be done not earlier than 
one year after radiotherapy. Patient is without the recurrence for 12 months but we know that any chemotherapy or radiotherapy will cause the huge toxicity that is why she is only under observation. We tried to balance the benefits from the radicality and the minimally invasive surgery at this particular patient.

\section{UNDIAGNOSED INVASIVE CERVICAL CANCER}

'J Amengual ${ }^{*},{ }^{1} \mathrm{~A}$ Torrent, ${ }^{2} \mathrm{AM}$ Quintero Duarte, ${ }^{1} \mathrm{M}$ Ruiz. ${ }^{1}$ University Hospital Son Espases, Gynaecological Oncology, Palma de Mallorca, Spain; ${ }^{2}$ University Hospital Son Espases, Anatomical Pathology, Palma de Mallorca, Spain

\subsection{6/ijgc-2021-ESGO.27}

Introduction/Background* Radical parametrectomy (RP) and upper vaginectomy (UV) is a challenging operation indicated when an occult cervical cancer (CC) is diagnosed after hysterectomy for another medical reason. It's a technically difficult procedure due to adhesions from the previous surgery and the absence of a uterus to assist dissection and achieve adequate negative margins.

Methodology

Result(s)* A 57-year-old woman, with no known medical comorbidities, was referred to our hospital from an outside private institution after having undergone a simple hysterectomy $(\mathrm{SH})$ with the diagnosis of incidental squamous cervical carcinoma.

The patient had initially presented with 4 weeks history of postmenopausal bleeding. No abnormalities were noted during a speculum examination, she had a negative smear test four months previously, and a normal pelvic ultrasound. Endometrial biopsy was inconclusive and the patient subsequently underwent a SH and bilateral salpingo-oophorectomy.

Pathology revealed an incidentally well differentiated squamous CC, measuring $3.5 \mathrm{~cm}$ in diameter and $1 \mathrm{~cm}$ of deep invasion (IB1 FIGO 2018). There was extensive lymphovascular space invasion and the cervical margins were also affected.

Postoperative staging scan (PET/TC), did not show any evidence of residual local or metastatic disease. A complete pelvic lymphadenectomy, with negative intraoperative result, RP and UV by laparoscopic-robotic surgery was performed. Due to the results of the LACC Trial, an open colpectomy was performed.

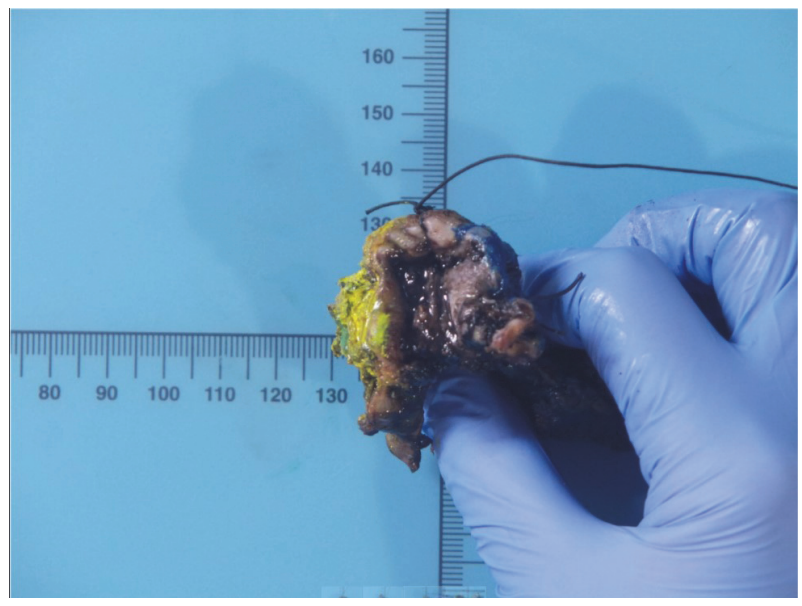

Abstract 385 Figure 1

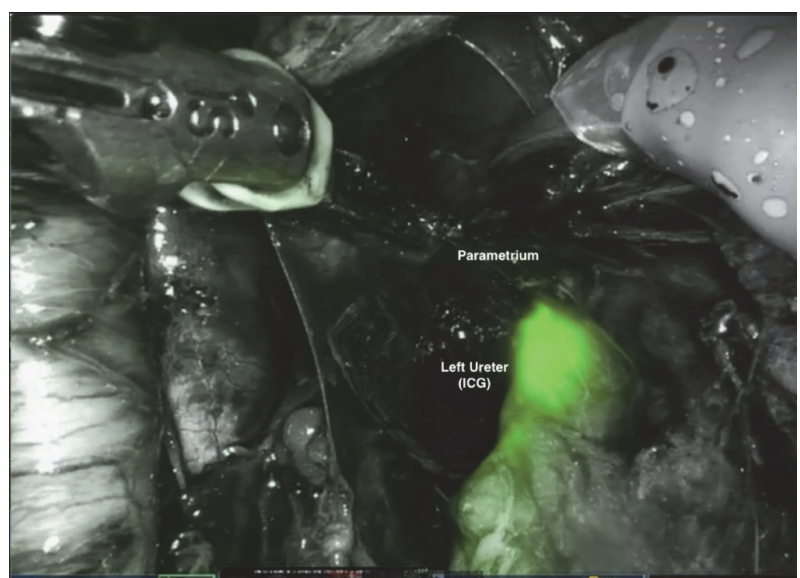

Abstract 385 Figure 2

The surgery lasted approximately $345 \mathrm{~min}$ and the patient was discharged 3 days after surgery. Two weeks later, she presented painful lymphoedema, and was diagnosed with bilateral pelvic lymphocysts, requiring drainage by interventional radiology.

Parametrectomy pathology demonstrated a residual focus (4 $\mathrm{mm}$ ) of squamous carcinoma at the vagina, and free margins. Subsequently, a metastasis was found in one left pelvic node upstaging to FIGO IIIC1. Adjuvant chemoradiation with weekly cisplatin and whole pelvic radiation was planned.

Conclusion* CC may be found incidentally after $\mathrm{SH}$ carried out for benign gynecologic conditions or preinvasive cervical lesions. $\mathrm{SH}$ is suboptimal procedure and associated with significantly inferior survival rates. Further treatment, such as radiotherapy (RT) or additional surgery, is warranted. PETCT have false negative, so surgery allow re-staging with a prognostic value and condition subsequent complementary treatment.

\section{SERIAL BONE DENSITY CHANGES IN WOMEN AFTER PELVIC CHEMORADIATION FOR CERVICAL CANCER}

${ }^{1} \mathrm{P}$ Chopade, ${ }^{2} \mathrm{~S}$ Chopra, ${ }^{2} \mathrm{~J}$ Jain, ${ }^{1} \mathrm{R}$ Engineer, ${ }^{2} \mathrm{~L}$ Gurram, ${ }^{1} \mathrm{P}$ Mittal, ${ }^{1} \mathrm{U}$ Mahantshetty, 2J Ghosh, 'S Gupta. 'Tata Memorial Hospital, Tata Memorial Centre., Radiation Oncology, Mumbai, India; ${ }^{2}$ Advanced Centre for Treatment Research and Education in Cancer, Tata Memorial Centre, Radiation Oncology, India

\subsection{6/ijgc-2021-ESG0.28}

Introduction/Background* Pelvic radiation therapy (RT) is associated with high doses to the lumbo-pelvic girdle. However, the impact of RT dose on bone density (BD) is not known. Present study was designed to understand the impact of RT dose on BD loss.

Methodology Patients recruited into a phase III trial of adjuvant radiation with at least 2 CT imaging data sets at baseline and follow up were eligible. The primary endpoint was to report correlation if any between RT dose and BD loss. Across the lumbopelvic region (L1-L5 vertebra, pubic symphysis, femur, acetabulum, greater trochanter, and anterior-superior iliac spine) points were predefined to estimate the RT dose received and Hounsfield (HU) units at pre RT and follow up time points on Eclipse version 13.5. Bone health was categorized as Normal $>130 \mathrm{HU}$, Osteopenic $=110-130 \mathrm{HU}$ or Osteoporotic $<110 \mathrm{HU}$ based on CT HU values. Univariate 